\title{
Stärkung von Bibliotheken in ländlichen Räumen
}

\section{Bund verlängert das Soforthilfeprogramm „Vor Ort für Alle“ des dbv für Bibliotheken in Kommunen bis 20.000 Einwohner*innen}

https://doi.org/10.1515/bd-2021-0069

Öffentliche Bibliotheken übernehmen in ländlichen Räumen wichtige Funktionen: Neben der Ausleihe von Medien bieten sie zunehmend auch Lernräume, Cafés, Maker Spaces, Co-Working-Plätze und Veranstaltungsorte. Damit schaffen sie eine wichtige Grundlage, um die kulturelle Versorgung der Bevölkerung in der digitalen Gesellschaft zu sichern.

Im Jahr 2020 wurden mit Hilfe des Soforthilfeprogramms „Vor Ort für Alle“ bereits 251 Bibliotheken in ländlichen Räumen bei ihrer Modernisierung und der Transformation zu Dritten Orten unterstützt. Auf Grund der großen Nachfrage hat sich der Deutsche Bibliotheksverband e.V. (dbv) für eine Verlängerung des Programms eingesetzt, die von Kulturstaatsministerin Monika Grütters unterstützt wird. Damit ist eine Fortführung des Programms im Jahr 2021 gesichert.

Hierdurch können Bibliotheken in Kommunen mit bis 20.000 Einwohner`innen ab dem Antragsstart am 20. Mai 2021 Fördermittel in Höhe von bis zu 25.000 Euro beim Deutschen Bibliotheksverband beantragen. Die Gesamtfördersumme beträgt 1,5 Millionen Euro. Das Programm ist auch für Nichtmitglieder des dbv offen.

Die Staatsministerin für Kultur und Medien: „Bibliotheken sind gerade in ländlichen Räumen weit mehr als Anlaufstellen, um Bücher und andere Medien auszuleihen. Sie sind vor allem auch lebendige Begegnungs- und Kulturorte. Nicht zuletzt in Zeiten der Corona-Pandemie wird uns allen bewusst, wie wichtig solche Angebote und Treffpunkte für die kulturelle Teilhabe und für den gesellschaftlichen Zusammenhalt sind. Daher setzen wir das Soforthilfeprogramm für Bibliotheken weiter fort und unterstützen noch mehr Einrichtungen dabei, insbesondere ihre digitale Ausstattung zu modernisieren."

Und Prof. Dr. Andreas Degkwitz, Bundesvorsitzender des Deutschen Bibliotheksverbandes, bekräftigt: „Gleichwertige Lebensverhältnisse von Stadt und Land sind keine Selbstverständlichkeit. Die große Überzeichnung des Soforthilfeprogramms im Jahr 2020 hat den Aufholbedarf sehr deutlich gemacht. Umso 
mehr freuen wir uns, auch in diesem Jahr im Rahmen von ,Vor Ort für Alle ‘ mehr kulturelle Teilhabe in ländlichen Räumen zu ermöglichen und Bibliotheken bei der Transformation zu Dritten Orten weiter zu unterstützen - zur Verbesserung der Lebensqualität aller dort lebenden Bürger*innen.“

\section{Vor Ort für Alle}

Das Soforthilfeprogramm „Vor Ort für Alle“ wird im Rahmen des Förderprogramms „Kultur in ländlichen Räumen“ von der Staatsministerin für Kultur und Medien gefördert aufgrund eines Beschlusses des Deutschen Bundestages. Die Mittel stammen aus dem Bundesprogramm „Ländliche Entwicklung“ (BULE) des Bundesministeriums für Ernährung und Landwirtschaft (BMEL).

Nähere Informationen zu dem Soforthilfeprogramm finden Sie unter: www. bibliotheksverband.de/dbv/projekte/vor-ort-fuer-alle.html.

\section{Der Deutsche Bibliotheksverband e.V. (dbv)}

Der Deutsche Bibliotheksverband e.V. (dbv) vertritt mit seinen fast 2.100 Mitgliedern bundesweit über 9.000 Bibliotheken mit 25.000 Beschäftigten und elf Millionen Nutzer^innen. Sein zentrales Anliegen ist es, Bibliotheken zu stärken, damit sie allen Bürger*innen freien Zugang zu Informationen ermöglichen. Der Verband setzt sich ein für die Entwicklung innovativer Bibliotheksleistungen für Wissenschaft und Gesellschaft. Als politische Interessensvertretung unterstützt der dbv die Bibliotheken insbesondere auf den Feldern Informationskompetenz und Medienbildung, Leseförderung und bei der Ermöglichung kultureller und gesellschaftlicher Teilhabe für alle Bürger`innen.

Mehr Informationen finden Sie unter www.bibliotheksverband.de.

\section{Pressekontakt}

Kristin Bäßler

Deutscher Bibliotheksverband

Leitung Kommunikation / Pressesprecherin

Tel.: + 49 (0)306449899 25 | E-Mail: baessler@bibliotheksverband.de

\section{Projektkontakt}

Mirko Winkelmann

Programmleiter „Vor Ort für Alle“

Tel.: + 49 (0)30644989919 | E-Mail: winkelmann@bibliotheksverband.de E-Mail: vorortfueralle@bibliotheksverband.de 
Gefördert durch:

Die Beauftragte der Bundesregierung
für Kultur und Medien

aufgrund eines Beschlusses des Deutschen Bundestages. 